\title{
Determinación de la causa de mortalidad en un vivero del langostino gigante de agua dulce Macrobrachium rosenbergii en Costa Rica: análisis de caso
}

\author{
DETERMINATION OF THE CAUSE OF MORTALITY IN A GIANT FRESHWATER PRAWN \\ Macrobrachium rosenbergii NURSERY IN COSTA RICA: CASE ANALYSIS
}

\author{
Alexander Varela-Mejías ${ }^{1,3}$, José Valverde-Moya ${ }^{2,4}$
}

\section{Resumen}

Se presentan los hallazgos obtenidos de una serie de análisis realizados para un laboratorio de maduración de langostinos de agua dulce de Macrobrachium rosenbergii en Costa Rica. En este centro de producción larval se presentaron abortos recurrentes de hembras grávidas, así como gran mortalidad en los estadios de larvas y pos-larvas. Los resultados indican que incrementos en la presencia de materia orgánica en el agua de los tanques causaron altas prevalencias de ectoparásitos en las masas ovígeras, así como elevados conteos bacteriales en la columna de agua. Esta condición generó cuadros de bacteriosis y parasitosis en las larvas con severas reducciones en la producción.

Palabras clave: langostinos de agua dulce; materia orgánica; parásitos; mortalidad

\section{Abstract}

This paper presents the findings obtained from a series of analyzes carried out for a freshwater prawn maturation of Macrobrachium rosenbergii in Costa Rica. A large larval production centre had recurrent abortions of gravid females, as well as high mortality in larval and post-larval stages. The results indicate that increases in the presence of organic matter in the water of the tanks caused high prevalence of ectoparasites in the ovigerous masses, as well as high bacterial counts in the water column. This condition generated bacterial and parasitic infections in larvae with severe reduction in production.

Key words: fresh water prawns; organic matter; parasites; mortality

\footnotetext{
${ }^{1}$ Laboratorio de Patologías y Parasitología de Crustáceos Oficializado por el Servicio Nacional de Salud Animal, Nicoya, Costa Rica

${ }^{2}$ Instituto Nacional de Aprendizaje, Núcleo Náutico Pesquero, Puntarenas, Costa Rica

${ }^{3}$ E-mail: alexander.varela@gmail.com

${ }^{4}$ E-mail: jvalverdemoy@ina.ac.cr
}

Recibido: 23 de octubre de 2017

Aceptado para publicación: 15 de marzo de 2018 


\section{INTRODUCCIÓN}

El langostino gigante de Malasia, también llamado langostino de agua dulce, Macrobrachium rosenbergii, presenta características que le permiten adaptarse con facilidad a condiciones para su producción en cautiverio. La adaptabilidad de esta especie facilita su cultivo en baja densidad de siembra y con relativamente poco nivel de tecnificación (Nandlal y Pickering, 2005; Marquez y MoraesValenti, 2012; Tidwell, 2012; Ahmed, 2013; Alam S y Alam M, 2014).

A pesar de ello, muchos viveros de langostinos de agua dulce han reducido sus niveles de producción de pos-larvas durante los últimos años, debido a un incremento en la incidencia de mortalidades masivas. Esto ha dado como resultado producciones inconsistentes, lo que se ha traducido en pérdidas económicas considerables (Briggs, 2013).

Costa Rica no ha sido la excepción en la reducción de la oferta de pos-larvas. El país pasó de producir 35 toneladas en 1999 a solo cinco toneladas en 2002; esto es, un descenso promedio de $53 \%$ anual en dicho periodo (FAO, 2004). Asimismo, la producción nacional de larvas ha sido estimada en cinco toneladas en 2005 (FAO, 2006). Por otro lado, en los últimos años, el principal centro de producción larval de $M$. rosenbergii en el país, Langostinos KoKo, ha venido presentando problemas severos con altas mortalidades y, por lo tanto, no ha sido capaz de cubrir las necesidades de semilla de los pequeños y medianos productores nacionales.

Las pérdidas económicas en este centro de producción larval se han estimado en cerca de US\$108 000 anuales, considerando que dejaron de producirse 1200 millares de pos-larvas, con un precio de US\$90.00 el millar. Además, se afectaron directamente las producciones de langostinos en estanques, debido a que estas han sido esporádicas y con rendimientos insuficientes por la falta de semilla.
Entre las principales causas de mortalidad en esta especie se encuentran los brotes de enfermedades en los estadios larvales, causados por bacterias, hongos, parásitos y virus (Jayaprakash et al., 2005; Gangnonngiw et al., 2009; Owens et al., 2009; DomínguezMachín et al., 2011; Corteel et al., 2012). En la mayoría de los brotes infecciosos predominan los causados por bacterias del género Vibrio (Jayaprakash et al., 2005). Asimismo, se han detectado enfermedades metabólicas causadas por desbalances en las dietas alimenticias (Kumar et al., 2004).

Los brotes infecciosos pueden ser detonados o exacerbados por factores ambientales adversos (Briggs, 2013; Hayd et al., 2014; Tiruvayipati y Bhassu, 2016). Este efecto ha sido reportado en camarones marinos (Lightner, 1996). Las fluctuaciones de parámetros ambientales influyen en las comunidades microbianas presentes $\mathrm{y}$, como resultado de ello, pueden proliferar especies patógenas (Cheng y Chen, 2004; Varela y Peña, 2013, 2015; Morales-Covarrubias y Gómez-Gil, 2014; Tiruvayipati y Bhassu, 2016).

Las mortalidades recurrentes reportadas por Langostinos KoKo conllevaron a la realización de una investigación por parte de un laboratorio especializado en diagnóstico de enfermedades de crustáceos. Por lo tanto, el objetivo de este estudio fue identificar la causa de las mortalidades, analizando las potenciales fuentes de contaminación y entrada de patógenos, con el fin de implementar acciones correctivas y medidas preventivas.

\section{Materiales y Métodos}

\section{Anamnesis del Caso}

El principal laboratorio de producción de larvas de M. rosenbergii en Costa Rica (Langostinos KoKo) ha venido presentando problemas severos y recurrentes de mortalida- 
des masivas de larvas y post larvas, así como abortos de masas ovígeras en los últimos dos años, lo que ha generado producciones con rendimientos inconsistentes e ineficientes.

Los principales parámetros del agua del laboratorio se han mantenido dentro de rangos aceptables, según Boyd y Zimmermann (2000) y Soundarapandian et al. (2009). Los registros de temperatura oscilan entre $28 \mathrm{y}$ $30{ }^{\circ} \mathrm{C}$, con no más de $1{ }^{\circ} \mathrm{C}$ de variación entre día y noche. La salinidad se regula a $12 \mathrm{~g} / \mathrm{l}$ durante todo el ciclo de cultivo. El pH fluctúa entre 7.5 y 8.0 y el oxígeno disuelto mantiene lecturas mayores a $5 \mathrm{mg} / 1$. La alcalinidad estuvo entre 80 y $130 \mathrm{mg} / 1 \mathrm{CaCO}_{3}$ y el contenido de cloro residual se registró a $0 \mathrm{mg} / \mathrm{l}$, al ser eliminado mediante aireación fuerte antes de utilizar el agua en los tanques de cultivo larval.

Metabolitos como el amoníaco y los nitritos se mantuvieron en $<0.1 \mathrm{mg} / 1$. Asimismo, como parte de los protocolos de rutina, se realiza la extracción de desechos desde el fondo de los tanques por succión, la limpieza diaria del tanque y el uso de filtros biológicos.

El laboratorio de maduración utiliza un tanque que recibe las aguas pretratadas mediante filtración y clorinación, donde se mantienen a las hembras y machos para el apareamiento y fecundación. En esta área, la hembra fecundada desova e incuba los huevos durante 18 días (Cavallo et al., 2001) hasta que eclosionen, para luego pasar por diferentes fases de desarrollo larval (VegaVillasante et al., 2011). Durante este tiempo están en contacto directo con el fondo del estanque, lugar donde hay acumulación evidente de detritus y materia orgánica.

\section{Cuadro Clínico}

La mortalidad de los animales se presenta en los estadios larvales y pos-larvales, pero, además, se detectan abortos de masas ovígeras en las hembras grávidas. Durante estos brotes, la mortalidad se presenta inme- diatamente después de la eclosión de los huevos o a la mitad del ciclo (8-10 días). En ocasiones no se detectan señales clínicas evidentes, los animales se tornan anoréxicos y las poblaciones desaparecen gradualmente.

Los signos clínicos son inespecíficos. Se observa la larva débil y moribunda, mostrando una conducta anormal con nadado débil, lento y errático, y se van al fondo del estanque por la poca actividad, reduciendo o interrumpiendo el consumo de alimento. Estas larvas pierden masa muscular y lucen anormalmente delgadas, presentando color pálido o blancuzco a grisáceo o verdoso. En algunos individuos se desarrollan manchas rojizas en el cuerpo por posible expansión de cromatóforos. Se presenta una reducción progresiva de la población en el tanque por mortalidad o canibalismo. Los animales no crecen y no mudan con normalidad. La mortalidad puede llegar al $100 \%$ de la población afectada dentro de los 2-3 días del inicio de los signos clínicos.

Como dato relevante adicional, se reporta que el estanque utilizado para reproducción no ha recibido vaciados sanitarios durante los últimos cinco años y ha estado sometido a uso continuo.

\section{Análisis de Laboratorio}

Se recolectaron cuatro muestras, conformadas por $1000 \mathrm{ml}$ de agua dulce, 1000 $\mathrm{ml}$ de agua salobre, masas ovígeras provenientes de tres hembras y 30 larvas sobrevivientes de un tanque con mortalidad severa. Las muestras fueron llevadas bajo refrigeración al Laboratorio de Patologías y Parasitología de Crustáceos en Nicoya, Costa Rica.

La mitad de la muestra de larvas (15 especímenes) y masas ovígeras fueron procesadas mediante análisis en fresco, siguiendo las metodologías descritas por DomínguezMachín et al. (2011). Además, se realizaron observaciones en fresco para la detección de epibiontes y parásitos, los cuales fueron iden- 
tificados y cuantificados por microscopía directa utilizando objetivos de 10x y 40x. Para el reporte de resultados se utilizó una escala progresiva en grados que van de G0 a G4, según el grado de severidad de las lesiones o daños que se detectan (Lightner, 1996; Valenti y Daniels, 2000). Además, se registra la presencia de parásitos, detritus y otras sustancias presentes, así como la respuesta del hospedador (melanización, inflamación, expansión de cromatóforos y otros).

En las necropsias, los 15 especímenes se disecaron tomando biopsias de cutícula, apéndices motores y orales, branquias, músculo esquelético, hepatopáncreas e intestino medio, los cuales fueron examinados al microscopio en forma individual. Los diferentes parásitos observados en las muestras fueron identificados en forma tentativa, basándose en la descripción morfológica de especies (Lightner, 1996; Varela y Peña, 2012; Mandal et al., 2015). Para la observación de las muestras se utilizó un microscopio compuesto Olympus $\mathrm{CH} 2$, sin utilizar tinciones o medios de contraste. Las imágenes de los hallazgos fueron capturadas mediante una cámara digital Canon SX50 HS.

Los análisis bacteriológicos se realizaron mediante cultivos en agar Tripticasa Soya (TSA) para bacterias totales aerobias y agar Tiosulfato-Citrato-Bilis-Sacarosa (TCBS), selectivo-diferencial para especies del género Vibrio. Las salinidades se ajustaron isotónicamente acorde al tipo de muestra. Las colonias más representativas aisladas en TCBS se purificaron y sometieron a identificación bioquímica, mediante el sistema API20NE, siguiendo las instrucciones del fabricante. Finalmente, se realizaron pruebas de sensibilidad a tres antibióticos: florfenicol, oxitetraciclina y enrofloxacina, mediante el método de difusión en agar Mueller-Hinton, siguiendo las metodologías descritas (Lightner, 1996; Hamed et al., 2003), aplicado a las

Cuadro 1. Resultados de análisis en fresco realizados a larvas de Macrobrachium rosenbergii

\begin{tabular}{|c|c|c|}
\hline Tejido / órgano & Observaciones & Grado \\
\hline Hepatopáncreas & $\begin{array}{l}\text { Los hepatopáncreas presentan reservas lipídicas normales, } \\
\text { ácinos bien conservados, no se detectan lesiones o efectos } \\
\text { citopáticos asociables a patógenos o parásitos. }\end{array}$ & G0 \\
\hline Intestino & $\begin{array}{l}\text { No se detectan lesiones o efectos citopáticos asociables a } \\
\text { patógenos o parásitos. }\end{array}$ & G0 \\
\hline Branquias & $\begin{array}{l}\text { Presencia moderada de detritus. No se detectan lesiones o } \\
\text { efectos citopáticos asociables a patógenos. Ectoparásitos } \\
\text { en lámelas branquiales, sin respuesta inflamatoria } \\
\text { asociada. }\end{array}$ & G1-2 \\
\hline Apéndices & $\begin{array}{l}\text { Presencia moderada de detritus. No se detectan lesiones o } \\
\text { efectos citopáticos asociables a patógenos. Acumulación } \\
\text { de parásitos, principalmente protozoos y, en menor grado, } \\
\text { bacterias filamentosas. }\end{array}$ & $\mathrm{G} 2$ \\
\hline Cutícula/músculo & $\begin{array}{l}\text { No se detectan lesiones o efectos citopáticos asociables a } \\
\text { patógenos. Presencia de ectoparásitos moderada, sin } \\
\text { lesiones o respuestas inflamatorias observables. }\end{array}$ & G1 \\
\hline Músculo & $\begin{array}{l}\text { No se detectan lesiones o efectos citopáticos asociables a } \\
\text { patógenos o parásitos. }\end{array}$ & G0 \\
\hline
\end{tabular}




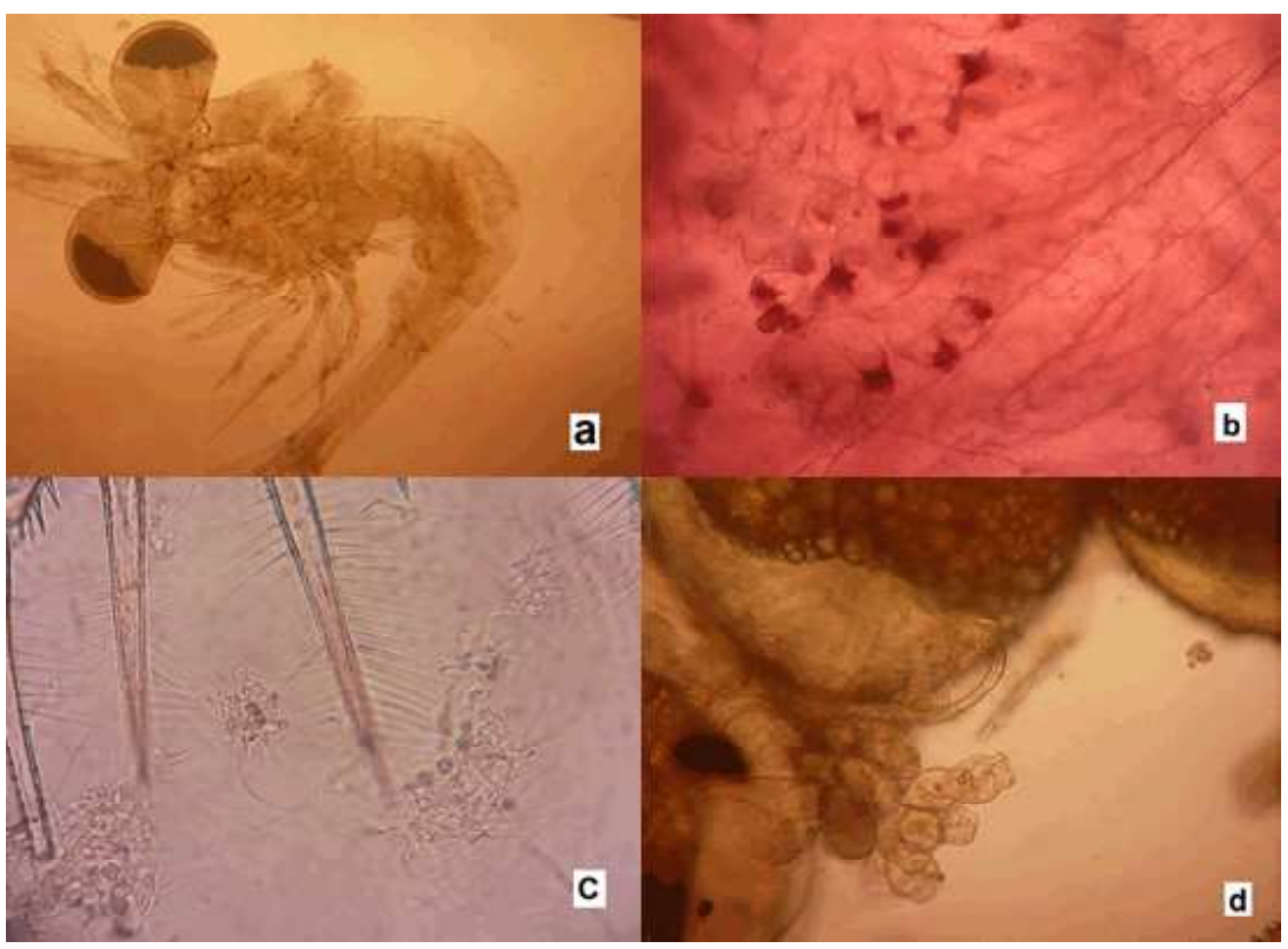

Figura 1. Microfotografías de tejidos de larvas de Macrobrachium rosenbergii. Sin tinción. a: cutícula con presencia moderada de epibiontes. 40X; b: lamela branquial con melanosis branquial apical diseminada. 400X; c: apéndice natatorio con acumulación de epibiontes. 400X: d: masa ovígera coloniza por epibiontes y acumulación de detritus. 400X

cepas de mayor patogenicidad según Lightner (1996) y Tiruvayipati y Bhassu (2016).

\section{Resultados}

Los hallazgos obtenidos en los órganos y tejidos de 15 larvas procesadas, así como los grados de severidad, se describen en el Cuadro 1. El hepatopáncreas, el intestino y el músculo presentaron condiciones aparentemente normales, sin lesiones o anomalías asociables a patógenos o parásitos. En la cutícula se detectó presencia moderada de parásitos, sin lesiones o respuestas inflamatorias asociables (Figura 1a). En las muestras de branquias se observó una acumulación moderada de detritus, con parasitosis por ciliados en lámelas branquiales sin respuesta inflamatoria asociada en la mayoría de los casos. Una de las muestras presentó melanosis branquial apical diseminada (Figura 1b). Los apéndices natatorios presentaron presencia moderada de detritus, sin lesiones asociadas a patógenos, pero con acumulación de parásitos, principalmente protozoos ciliados (Figura 1c).

Por su parte, en las muestras de masas ovígeras se detectó una elevada colonización por parásitos, especialmente ciliados peritricos, y en menor grado bacterias filamentosas. Además, una importante acumulación de detritus asociado a cúmulos de bacterias filamentosas, sin lesiones detectables asociadas (Figura 1d). 
Cuadro 2. Conteo de bacterias en huevos y larvas de Macrobrachium rosenbergii y en muestras de agua de los tanques del centro de producción larval (Costa Rica)

\begin{tabular}{llccccc}
\hline \multirow{2}{*}{ Muestra } & Mx & Agar TSA & \multicolumn{3}{c}{ Agar TCBS } & Salinidad \\
\cline { 3 - 5 } & Totales & Sacaroliticas & No sacaroliticas & $\begin{array}{c}\text { Bio- } \\
\text { luminiscentes }\end{array}$ & \\
\hline $\begin{array}{l}\text { AV-2017- } \\
0047\end{array}$ & $\begin{array}{l}\text { Agua } \\
\text { dulce }\end{array}$ & 900 & $<100$ & $<100$ & $<100$ & 0 \\
$\begin{array}{l}\text { AV-2017- } \\
0048\end{array}$ & $\begin{array}{l}\text { Agua } \\
\text { salada }\end{array}$ & 4,300 & 2,400 & 100 & $<100$ & 12 \\
$\begin{array}{l}\text { AV-2017- } \\
0049\end{array}$ & $\begin{array}{l}\text { Masa } \\
\text { ovigera }\end{array}$ & 14,600 & 3,800 & 300 & $<100$ & \\
$\begin{array}{l}\text { AV-2017- } \\
0050\end{array}$ & Pos-larva & 27,400 & 5,800 & 900 & 300 & \\
\hline
\end{tabular}

Cuadro 3. Caracterización e identificación de bacterias aisladas en agar Tiosulfato-CitratoBilis-Sacarosa (TCBS) en huevos y larvas de Macrobrachium rosenbergii en un centro de producción con alta mortalidad (Costa Rica)

\begin{tabular}{|c|c|c|c|c|}
\hline \multicolumn{5}{|c|}{ Descripción fenotípica de las colonias } \\
\hline Muestra & Consecutivo & Colonia & \multicolumn{2}{|c|}{ Descripción de colonia } \\
\hline \multirow[t]{2}{*}{ Huevos } & \multirow{2}{*}{$\begin{array}{l}\text { AV-2017- } \\
0049\end{array}$} & $\mathrm{C} 1$, Sacarolítica & \multicolumn{2}{|c|}{ Convexa, circular, regular, mediana } \\
\hline & & C2, No sacarolítica & \multicolumn{2}{|c|}{$\begin{array}{l}\text { Convexa, circular, regular, muy } \\
\text { pequeña }\end{array}$} \\
\hline \multirow[t]{2}{*}{$\begin{array}{l}\text { Pos- } \\
\text { larvas }\end{array}$} & \multirow[t]{2}{*}{$\begin{array}{l}\text { AV-2017- } \\
0500\end{array}$} & C3, No sacarolítica & \multicolumn{2}{|c|}{$\begin{array}{l}\text { Convexa, circular, regular, muy } \\
\text { pequeña }\end{array}$} \\
\hline & & C4, No sacarolítica & \multicolumn{2}{|c|}{$\begin{array}{l}\text { Convexa, circular, regular, muy } \\
\text { pequeña, bioluminiscente }\end{array}$} \\
\hline \multicolumn{5}{|c|}{ Identificación basada en el perfil bioquímico } \\
\hline \multirow[t]{4}{*}{ Cepas } & \multicolumn{2}{|l|}{$\mathrm{C} 1$} & \multicolumn{2}{|c|}{ Vibrio alginolyticus } \\
\hline & \multicolumn{2}{|l|}{$\mathrm{C} 2$} & \multicolumn{2}{|c|}{ Vibrio parahaemolyticus } \\
\hline & \multicolumn{2}{|l|}{ C3 } & \multicolumn{2}{|c|}{ Vibrio parahaemolyticus } \\
\hline & $\mathrm{C} 4$ & & \multicolumn{2}{|c|}{ Vibrio harveyi } \\
\hline \multicolumn{5}{|c|}{ Resultados de las pruebas de sensibilidad a antibióticos } \\
\hline \multirow{5}{*}{ Cepas } & & Enrofloxacina & Florfenicol & Oxitetraciclina \\
\hline & $\mathrm{C} 1$ & $\mathrm{~S}$ & $\mathrm{~S}$ & $\mathrm{~S}$ \\
\hline & $\mathrm{C} 2$ & $\mathrm{~S}$ & $\mathrm{~S}$ & $\mathrm{R}$ \\
\hline & $\mathrm{C} 3$ & $\mathrm{~S}$ & S & $\mathrm{R}$ \\
\hline & $\mathrm{C} 4$ & S & I & I \\
\hline
\end{tabular}

Vibrio parahaemolyticus y $V$. harveyi son consideradas patogénicas

$\mathrm{S}$ : sensible. I: intermedia. R: resistente 
No se observaron esporas o hifas que indiquen cuadros micóticos. Tampoco hubo lesiones sugerentes de infecciones virales, $\mathrm{y}$ aunque se debe considerar la limitada sensibilidad del método de análisis utilizado en las muestras, no hay sospechas clínicas de virosis, ni se identificaron nexos epidemiológicos sustentables.

Los resultados del análisis bacteriológico de las muestras de agua dulce, agua sala$\mathrm{da}$, larvas enfermas y masas ovígeras se presentan en el Cuadro 2. Se observan conteos elevados de bacterias en masas ovígeras y larvas, con marcado predominio de especies sacarolíticas. La presencia de especies bioluminiscentes se corroboró únicamente en la muestra de larvas. Por su parte, las muestras de aguas presentaron presencia bacterial aeróbica, y presencia de especies del género Vibrio en la muestra de agua salobre.

Información adicional de las bacterias, incluyendo la forma de las colonias que conforman las bacterias recuperadas en el agar TCBS, la reacción a Gram, e identidad basada en el perfil bioquímico, así como su sensibilidad a los antibióticos ensayados se describe en el Cuadro 3. La mayor sensibilidad se presentó a la Enrofloxacina.

\section{Discusión}

Las observaciones macroscópicas realizadas durante la visita al laboratorio de Langostinos KoKo evidenciaron acumulaciones de detritus y materia orgánica en las masas ovígeras, portadas por hembras provenientes del estanque de reproductores. Este también presentaba una importante acumulación de materia orgánica. Asimismo, las larvas se encontraban expuestas a condiciones subóptimas, que facilitan y promueven las parasitosis detectadas.

Tanto en las muestras de masas ovígeras como en las larvas, la presencia de parásitos fue constante. En condiciones de baja colo- nización, estos organismos no son considerados un problema serio, y las infestaciones severas suelen estar más estrechamente relacionadas a la cantidad de la materia orgánica en el agua o el organismo. Sus colonias generalmente están formadas por poblaciones de protozoos epicomensales como Epistylis sp, Zoothamnium sp, Acineta sp y Vorticella sp y bacterias filamentosas como Leucothrix molitrix (Mandal et al., 2015). Cuando las infestaciones se presentan en grandes cantidades pueden ser fuente de estrés y potencial asfixia, al reducir la eficiencia del intercambio gaseoso en branquias. Estas condiciones generan bajo crecimiento, obstrucción de los procesos de muda y posibles mortalidades (Pillai y Bonami, 2012).

Los resultados bacteriológicos sugieren deficiencias importantes en los procesos de desinfección del agua marina, la cual presentaba cepas de Vibrio, aun posterior a la clorinación. Estas bacterias también estaban presentes en las larvas y en las masas ovígeras, sugiriendo que los tanques deben presentar conteos importantes de bacterias totales y de Vibrio sp.

La cantidad de bacterias totales recuperadas en las masas ovígeras fue considerable, al tiempo que se presentaron conteos moderados de Vibrio sp. La presencia de bacterias no sacarolíticas en masas ovígeras, larvas y pos-larvas se considera potencialmente peligrosa, debido a que en este grupo se encuentran especies bioluminiscentes como $V$. harveyi y $V$. parahaemolyticus, así como otras que, sin ser bioluminiscentes, son altamente patógenas para los crustáceos (Morales-Covarrubias y Gómez-Gil, 2014; Tiruvayipati y Bhassu, 2016; Varela-Mejías et al., 2017).

Las infecciones bacterianas a inicios del ciclo son causadas, por lo general, por especies del género Vibrio. Además de $V$. parahaemolyticus y $V$. harveyii, pueden coexistir otro tipo de bacterias menos virulentas como $V$. alginolyticus, Staphylococcus sp, Streptococcus sp y Micrococcus 
$\mathrm{sp}$, actuando como patógenos oportunistas, así como algunas especies quitinolíticas, entre ellas Pseudomonas y Aeromonas. No se realizaron análisis para especies intracelulares como las Rickettsia (Cheng y Chen, 2004; Phatarpekar et al., 2008), debido a que no son cultivables en agares sintéticos y, por tanto, no detectables mediante los ensayos realizados.

Los cuadros de infecciones por bacterias se controlan usualmente con antibióticos de amplio espectro (Pillai y Bonami, 2012). Las pruebas de sensibilidad a antibióticos indicaron que la mejor opción terapéutica era la enrofloxacina; sin embargo, los resultados no fueron los esperados para ese evento y las mortalidades continuaron. Es posible que esta ineficacia terapéutica se explique por el hecho de estar ante un lote con estado infeccioso avanzado.

Con la integración de los resultados de análisis en fresco y bacteriología, mostrando altos conteos bacteriales, parásitos y detritus, se concluye que el problema principal se origina en un ineficiente filtrado y tratamiento de aguas para los tanques, lo cual es consistente con la detección de bacterias Vibrio en muestras de agua salada, posterior a las clorinaciones. Esto estaría indicando ineficacia del proceso de sanitización. Las acumulaciones de detritus por su parte indican que la extracción de sedimentos por succión no está eliminando la totalidad de esta materia.

Finalmente, a pesar de no lograrse la recuperación del lote larval analizado, se logró identificar la etiología de las mortalidades y su fuente. Con base en los resultados del estudio, se implementaron una serie de acciones correctivas para evitar reincidencias en los lotes subsiguientes. Las acciones correctivas estuvieron enfocadas principalmente sobre los sistemas de filtración y tratamiento de agua de mar, así como en la aplicación de desinfección, vaciado sanitario, secado, arado y encalado del estanque de reproducción, el cual se sometió a un periodo de descanso.

\section{Literatura Citada}

1. Ahmed N. 2013. On-farm feed management practices for giant river prawn (Macrobrachium rosenbergii) farming in southwest Bangladesh. In: Hasan MR, New MB (eds). On-farm feeding and feed management in aquaculture. FAO Fisheries and Aquaculture Technical Paper N. ${ }^{\circ} 583$. Rome: FAO. p 269-301.

2. Alam S, Alam M. 2014. Development of the giant freshwater prawn Macrobrachium rosenbergii (De Man 1779) broodstock in culture ponds of South-Western Bangladesh: a case study. J Entomol Zool Studies 2(5): 108113.

3. Boyd C, Zimmermann S. 2000. Growout systems - Water quality and soil management. In: New M, Valenti W (eds). Freshwater prawn culture: the farming of Macrobrachium rosenbergii. London: Blackwell Science. p 221238.

4. Briggs M. 2013. Analysis of freshwater prawn hatchery problems in Bangladesh. Report to USAID funded. Feed the Future Aquaculture (FtF AQ) project of the World Fish Center, Bangladesh and South Asia Office. 43 p.

5. Cavallo $R$, Lavens P, Sorgeloos P. 2001. Reproductive performance of Macrobrachium rosenbergii females in captivity. J World Aquac Soc 32: 60-67. doi: 10.1111/j.1749-7345.2001.tb00922.x

6. Cheng W, Chen JC. 2004. Enterococcus-like infections in Macrobrachium rosenbergii are exacerbated by high $\mathrm{pH}$ and temperature but reduced by low salinity. Dis Aquat Org 34: 103-108.

7. Corteel M, Dantas-Lima JJ, Tuan VV, Thuong VK, Wille M, Alday-Sanz V, Pensaert MV, et al. 2012. Susceptibility of juvenile Macrobrachium rosenbergii to different doses of high and low virulence strains of white spot syndrome virus (WSSV). Dis Aquat Organ 100: 211-218. doi: 10.3354/dao02496 
8. Domínguez-Machín ME, HernándezVergara MP, Jiménez-García I, SimáAlvarez, R, Rodríguez-Canul R. 2011. Survey of protozoan, helminth and viral infections in shrimp Litopenaeus setiferus and prawn Macrobrachium acanthurus native to the Jamapa River region, Mexico. Dis Aquat Organ 96: 97103. doi: $10.3354 /$ dao02392

9. [FAO] Organización de las Naciones Unidas para la Alimentación y la Agricultura. 2004. Perfiles sobre la pesca y la acuicultura por países. La República de Costa Rica. [Internet]. Disponible en: http://www.fao.org/ fishery/facp/CRI/es\#CountrySectorOverview

10. [FAO] Organización de las Naciones Unidas para la Alimentación y la Agricultura. 2006. Visión general del sector acuícola en Costa Rica. Departamentos de Pesquería y acuicultura de la FAO y del INCOPESCA. [Internet]. Disponible en: http://www.fao.org/ fishery/countrysector/naso_costarica/es

11. Gangnonngiw W, Kiatpathomchai $W$, Sriurairatana $S$, Laisutisan $K$, Chuchird N, Limsuwan C, Flegel TW. 2009. Parvo-like virus in the hepatopancreas of freshwater prawns Macrobrachium rosenbergii cultivated in Thailand. Dis Aquat Organ 85: 167173. doi: $10.3354 /$ dao02075

12. Hamed A, Rahaman V, Alagan A, Yoganandhan K. 2003. Antibiotic resistance to bacteria aisolated from hatchery-reared larvae and post-larvae of Macrobrachium rosenbergii. Aquaculture 217: 39-48. doi: 10.1016/ S0044-8486(02)00298-3

13. Hayd L, Lemus D, Valenti W. 2014. Effects of ambient nitrite on Amazon River prawn, Macrobrachium amazonicum, larvae. J Word Aqua Soc 45: 55 64. doi: 10.1111/jwas. 12071

14. Jayaprakash NS, Pai S, Anas A, Preetha R, Philip R, Singh IS. 2005. A marine bacterium, Micrococcus MCCB 104, antagonistic to vibrios in prawn larval rearing systems. Dis Aquat Organ 68: 39-45. doi: 10.3354/dao068039
15. Kumar AR, Rao GV, Rao KR. 2004. Appendage deformity syndrome - a nutritional disease of Macrobrachium rosenbergii. Dis Aquat Organ 59: 7578. doi: $10.3354 /$ dao0 059075

16. Lightner DV. 1996. A handbook of pathology and diagnostic procedures for diseases of cultured penaeid shrimp. USA: World Aquaculture Society. 304 p.

17. Mandal B, Dubey S, Ghosh A, Dash G. 2015. Parasitic occurrence in the giant freshwater prawn Macrobrachium rosenbergii from coastal West Bengal, India. J Parasitol Vector Biol 7(6): 115-119.

18. Marquez, H, Moares-Valenti P. 2012. Review article: Current status and prospects of farming the giant river prawn (Macrobrachium rosenbergii (De Man, 1879) and the Amazon river prawn Macrobrachium amazonicum (Heller 1862) in Brazil. Aquac Res 43: 984-992. doi: 10.1111/j.13652109.2011.03032.x

19. Morales-Covarrubias MS, Gómez-Gil B. 2014. Enfermedades bacterianas de camarones. En: Morales V, Cuéllar-Anjel J (eds). Guía técnica: patología e inmunología de camarones penaeidos, $2^{\circ}$ ed. Rep. de Panamá: OIRSA. p 167-192.

20. Nandlal S, Pickering T. 2005. Freshwater prawn Macrobrachium rosenbergii farming in Pacific Island countries. Vol I. Hatchery operation. Noumea, New Caledonia: Secretariat of the Pacific Community. $31 \mathrm{p}$.

21. Owens L, La Fauce K, Juntunen K, Hayakijkosol O, Zeng C. 2009. Macrobrachium rosenbergii nodavirus disease (white tail disease) in Australia. Dis Aquat Organ 85: 175-180. doi: 10.3354/dao02086

22. Phatarpekar P, Kenkre V, Sreepada R, Dasai V, Achuthankutty C. 2008. Bacterial flora associated with larval rearing of the giant freshwater prawn Macrobrachium rosenbergii. Aquaculture 203: 279-291. doi: 10.1016/ S0044-8486(01)00705-0 
23 Pillai D, Bonami J. 2012. A review on the diseases of freshwater prawns with special focus on white tail disease of Macrobrachium rosenbergii. Aquac Res 43: 1029-1037. doi: 10.1111/j.13652109.2011.03061.x

24 Tidwell J. 2012. Current status and prospects of farming the giant river prawn (Macrobrachium rosenbergii De Man 1879) in the United States. Aquac Res 43: 1023-1028. doi: 10.1111/ j.1365-2109.2012.03130.x

25 Tiruvayipati S, Bhassu S. 2016. Host, pathogen and the environment: the case of Macrobrachium rosenbergii, Vibrio parahaemolyticus and magnesium. Gut Pathog 8: 15. doi: 10.1186/s13099-0160097-1

26 Valenti W, Daniels W. 2000. Recirculation hatchery systems and management. In: New M, Valenti W (eds). Freshwater prawn culture: the farming of Macrobrachium rosenbergii. London: Blackwell Science. p 69-90.

27 Varela A, Peña N. 2012. Efecto de los epibiontes en los sistemas productivos de- camarón en Costa Rica. Revista UTN Informa 62: 70-73.

28 Varela A, Peña N. 2013. El virus del síndrome de las manchas blancas (WSSV): una revisión y su impacto en la camaronicultura costarricense. Rev Cienc Vet 28(2): 51-69.

29 Varela A, Peña N. 2015. Hepatopancreatitis necrotizante asociada al Fenómeno del Niño, en cultivos de camarones del Golfo de Nicoya. Repertorio Científico18(1): 29-34.

30 Varela-Mejía A, Peña-Navarro N, Aranguren-Caro LF. 2017. Necrosis aguda del hepatopáncreas: una revisión de la enfermedad en Penaeus vannamei. Agron Mesoam 28: 735-745. doi: 10.15517/ma.v28i3.27788

31 Vega-Villasante F, Espinosa L, Granados $S$, Cortés E, García M, Leví A, Nolasco H, Guzmán M. 2011. Acuicultura del langostino Macrobrachium tenellum: engorda en estanques semi rústicos. México: Univ. de Guadalajara. $87 \mathrm{p}$. 